\title{
C-MYC and BCL2: Correlation between Protein Over-Expression and Gene Translocation and Impact on Outcome in Diffuse Large B Cell Lymphoma
}

\author{
Amrallah A Mohammed ${ }^{1 *}$, Hayam E Rashed ${ }^{2}$, Aziza E Abdelrahman', \\ Ahmed A Obaya ${ }^{3}$, Mostafa Toam ${ }^{3}$, Hanim M Abdel Nour ${ }^{4}$, Mohamed I \\ Abdelhamid $^{5}$, Fifi Mostafa Elsayed ${ }^{6}$
}

\begin{abstract}
Background: Due to lack of availability of gene expression profiling $(G E P)$ for most developing countries and clinicians; the immunohistochemistry (IHC) is mostly used in the clinical application. The aim of our study is to check the possibility of using IHC to detect MYC and BCL2 in our patients with diffuse large B-cell lymphoma (DLBCL) instead of GEP to stratify them into high and low-risk groups. This will help in a proper treatment choice of subsequent improvement in the survival outcome. Method: During the study period, 90 DLBCL patients were eligible. MYC and BCL2 evaluated by IHC and gene rearrangement by real-time PCR (RT-PCR) and correlated with clinical-pathological features and survival. Results: Through IHC, the expression of MYC, BCL2, and double expression was detected in $35.6 \%, 46.7 \%$ and $30 \%$ of patients, respectively. While by RT-PCR, it was $4.53 \pm 0.74$ for MYC compared with $2.18 \pm 0.78$ for BCL-2. Most patients with BCL2+/MYC+; double-expressor and double-hit lymphomas (DEL and DHL) had high stage (III, IV), more extra-nodal involvement, (P value $<0.001)$ and intermediate to high International Prognostic Index (IPI) risk profile (P-value $<0.001$ ). The median overall survival was 14 months and 6 months for DEL and DHL, respectively. While all patients with DHL died during the follow-up period, the median PFS were only 2 months for DEL. There was a statistically significant correlation between mRNA of MYC and BCL2 with their protein expression $(\mathrm{p}<0.001)$. Conclusion: Our results confirmed the unique characters and poor outcome associated with DEL and DHL mandated the need for more intense therapy and not the standard protocol. Moreover, the significant correlation between protein overexpression and gene rearrangement may open the door for the possibility to use IHC instead of RT-PCR in developing countries.
\end{abstract}

Keywords: Double expressor lymphoma- double hit lymphoma- Immunohistochemistry- MYC- BCL2- RT-PCR

Asian Pac J Cancer Prev, 20 (5), 1463-1470

\section{Introduction}

Diffuse large B-cell lymphoma (DLBCL) is a heterogeneous clinical, pathological, immunophenotypic, and genetic disease. Based on gene signatures and cell of origin ( $\mathrm{COO})$, it is classified into germinal center B-cell (GCB)-like with favorable prognosis and unfavorable activated B-cell (ABC)-like phenotypes (Barrans et al., 2012).

Double-hit lymphomas $(D H L)$ are a subtype of $D L B C L$, characterized by translocations involving the $M Y C$ gene combined with either translocation of the BCL2 or BCL6 gene. When the three translocations occurred and detected at the same time called triple-hit lymphomas (THL). Whereas, detection of protein overexpression (not gene translocation), is called double-expressor lymphoma (DEL).

The diagnosis of DHL is only determined following the results of a cytogenetic test, such as fluorescence in situ hybridization (FISH). They comprise $15 \%$ of B-cell lymphoma with clinical features intermediate between DLBCL and Burkitt's lymphoma (BL). While DEL accounted for $20 \%$ to $30 \%$ (Jaffe et al., 2008; Aukema et al., 2011).

The World Health Organization (WHO) updated to recognize the co-expression of $M Y C$ and $B C L 2$ proteins as a new adverse prognostic marker. In case of DHL or DEL, the prognosis is poor after the standard chemotherapy protocol, R-CHOP (cyclophosphamide, doxorubicin, vincristine, prednisone, and Rituximab chemotherapy)

${ }^{1}$ Department of Medical Oncology, ${ }^{2}$ Department of Pathology, ${ }^{3}$ Department of Clinical Oncology, ${ }^{4}$ Department of Biochemistry, ${ }^{5}$ Department of General Surgery, Faculty of Medicine, Zagazig University, ${ }^{6}$ Department of Clinical Oncology and Nuclear Medicine, Faculty of Medicine, Suez Canal University, Egypt.*For Correspondence: amrallaabdelmoneem@yahoo.com 
with less than 30\% long-term survivors (Hu et al., 2013).

Currently, the best treatment regimen for DHL is unknown, however, more intensive treatment protocols such as EPOCH-R (etoposide, prednisone, vincristine, cyclophosphamide, and doxorubicin plus Rituximab) may give better results than the standard one (Oki et al., 2014).

IHC is the most significant supplementary tool for the evaluation of lymphoma because of its practicability and low cost. In contrast, molecular cytogenetic techniques are expensive and not available in most clinical laboratories.

The aim of this study is to evaluate the association of protein overexpression and gene translocation of $M Y C$ and $B C L 2$ in $D L B C L$ and also to address the correlation with clinical-pathological features and survival outcome.

\section{Materials and Methods}

Patients, study design, and ethical aspect

The present retrospective study included 90 de novo DLBCL patients diagnosed at the Pathology Department, Zagazig University during the period from January 2011toMarch 2015. They received the classic management, R-CHOP at Medical Oncology and Clinical Oncology Departments. The diagnosis was based on the 2016 WHO classification criteria (Swerdlow, 2016). By the use of medical files of patients; the demographic data, clinical-pathological features, laboratory workup, and follow up period were recorded retrospectively. The study included 15 lymphoid tissue samples from reactive lymph nodes in age and sex-matched patients which represented control samples. All specimens were divided into two parts; the first one was frozen at $-80^{\circ} \mathrm{C}$ until used for detection of $M Y C$ and BCL2 gene expression by RT-PCR in Medical Biochemistry Department. The second was immediately fixed in $10 \%$ formalin for histopathologic examination in the Pathology Department, Zagazig University.

The patient's data and names were protected without patient identifiers. The institutional review board (IRB) approved the study.

\section{Histopathology and IHC}

Hematoxylin and eosin ( $\mathrm{H}$ and $\mathrm{E}$ ) stained slides were evaluated. IHC analysis was performed using the polymer Envision detection system; the Dako EnVision тм kit (Dako, Copenhagen, Denmark). Antibodies that used were: $C D 20$ (clone L26, Dako, Carpinteria, CA), CD79a (clone JCB117, Dako, ready to use), CD10 (clone 56C6, ready to use, Dako), Bcl6 ( $P G B 6-P$ clone, ready to use, Dako), Bcl2 (clone 124, ready to use, Dako), and Myc (clone EP121, ready to use, Biocare). Diaminobenzidine was used as the chromogen and hematoxylin as the counterstaining.

\section{IHC assessment}

The expressions of $C D 20, C D 79 a$, and $C D 10$ were assessed for positivity or negativity. The WHO classification defines over-expression of $M Y C$ protein $\geq 40 \%, B C L 2$ protein $\geq 50 \%$ (Hu et al., 2013), and Ki67 index $\geq 90 \%$ (Tang et al., 2017).
RT-PCR analysis for MYC and BCL2 gene expression $R N A$ and $c D N A$ preparation

Total RNA was isolated from tissue samples using RNeasy (Qiagen, Valencia, CA) according to the manufacturer's instructions. The reverse transcription reaction was done using a reverse transcription kit (Reverse Transcriptase, Roche Diagnostics) following the manufacturer's protocol.

\section{Quantitative RT-PCR}

$M Y C$ and $B C L 2$ expressions were detected as previously described by Xia et al., (2015). $\beta$-actin was used as a reference gene. Amplification for MYC and $B C L-2$ were performed in a total volume of $20 \mu \mathrm{L}$ containing $10 \mu \mathrm{L}$ of kit-supplied QuantiTect ${ }^{\mathrm{TM}}$ SYBR ${ }^{\circledR}$ Green RT-PCR Master mix (Applied-Biosystems), $0.4 \mu \mathrm{L}$ of each primer, $2 \mu \mathrm{L}$ of $\mathrm{cDNA}$ and $7.2 \mu \mathrm{L}$ $\mathrm{ddH}_{2} \mathrm{O}$. Primer pair sequences for the $M Y C$ gene were; c-Myc-F: CCTCCACTCGGAAGGACTATC; c- $M y c-\mathrm{R}$ : TGTTCGCCTCTTGACATTCTC, for BCL-2 were; BCL2-F; GTGGATGACTGAGTACCTGAACC; BCL-2-R: AGACAGCCAGGAGAAATCAAAC and for $\beta$-Actin were $\beta$-Actin-F: CCTGGCACCCAGCACAAT; $\beta$-Actin-R: GGGCCGGACTCGTCATAC. The PCR cycling parameters were set as follows: $95 \mathrm{C}$ for $30 \mathrm{~s}$ followed by 40 cycles of PCR reaction $95{ }^{\circ} \mathrm{C}$ for $5 \mathrm{~s}$ and $60^{\circ} \mathrm{C}$ for $34 \mathrm{~s}$. The amplification was carried out using Real-time PCR (StratageneMx3005P-qPCR System). Relative changes in gene expression were calculated using the $2^{-\Delta \Delta \mathrm{CT}}$ method (Livak and Schmittgen 2001).

\section{Treatment protocol and response evaluation}

The eligible patients followed the chart as illustrated in Figure 1A.

\section{Statistical Analysis}

All statistics were performed using SPSS 22.0 for Windows (SPSS Inc., Chicago, IL, USA) and MedCalc windows (MedCalc Software bvba 13, Ostend, Belgium). Parametric and non-parametric t-tests were used for comparison of two independent groups. Progression-free survival (PFS) was defined as the time interval from the first diagnosis until disease progression, while overall survival (OS) was defined as the time from the initial diagnosis to death or the last follow up. PFS and OS were estimated according to Kaplan-Meier and compared by the log-rank test. Multivariate analyses were performed with the use of a Cox regression model to estimate hazard ratios for an evolving event. All $\mathrm{P}$ values are based on 2-tailed statistical analysis, considering $\mathrm{P}$ values $<0.05$ as significant.

\section{Results}

\section{Patients and clinical-pathological parameters}

Of the 90 patients; 49 were males with a mean age $57.82 \pm 14.11$ (range 25-90). Extra-nodal involvement was observed in about $29 \%$ of cases. According to the Ann Arbor Staging system, stage I included 14 patients, stage II $(n=25)$, stage III $(n=35)$ and stage IV $(n=16)$. All cases were DLBCL with diffuse growth pattern, 
Table1. Clinical-pathological Features, Immunohistochemical Markers and Outcome in Studied Patients with NHL.

\begin{tabular}{llcc}
\hline Characteristics & \multicolumn{2}{c}{$\begin{array}{c}\text { All patients }(\mathrm{N}=90) \\
\text { No. }\end{array}$} \\
\hline Age & Mean \pm SD & \multicolumn{2}{c}{$57.82 \pm 14.11$} \\
(Years) & Median (Range) & \multicolumn{2}{c}{$58(25-90)$} \\
Sex & Male & 49 & $(54.4)$ \\
& Female & 41 & $(45.6)$
\end{tabular}

Extranodal involvement

\begin{tabular}{|c|c|c|c|}
\hline Absent & & 64 & $(71.1)$ \\
\hline Present & & 26 & $(28.9)$ \\
\hline Stage & Stage I & 14 & $(15.6)$ \\
\hline & Stage II & 25 & $(27.8)$ \\
\hline & Stage III & 35 & $(38.9)$ \\
\hline & Stage IV & 16 & $(17.8)$ \\
\hline IPI risk group & Low & 14 & $(15.6)$ \\
\hline & Low-Intermediate & 23 & $(25.6)$ \\
\hline & High-Intermediate & 33 & $(36.7)$ \\
\hline & High & 20 & $(22.2)$ \\
\hline Response & PD & 27 & -30 \\
\hline & $\mathrm{SD}$ & 15 & $(16.7)$ \\
\hline & $\mathrm{PR}$ & 28 & $(31.1)$ \\
\hline & $\mathrm{CR}$ & 20 & $(22.2)$ \\
\hline & OAR & 48 & $(53.3)$ \\
\hline & NR & 42 & $(46.7)$ \\
\hline *Follow-up & Mean \pm SD & 24. & .38 \\
\hline & Median (Range) & & \\
\hline Relapse & & $(\mathrm{N}=2 \mathrm{C}$ & \\
\hline & Before 24 month & 8 & -40 \\
\hline & After 24 month & 12 & -60 \\
\hline Progression & & $(\mathrm{N}=42$ & \\
\hline & Within 6 month & 30 & $(71.4)$ \\
\hline & After 6 month & 12 & $(28.6)$ \\
\hline Mortality & A live & 66 & $(73.3)$ \\
\hline & Died & 24 & $(26.7)$ \\
\hline Ki 67 & Low & 23 & $(25.6)$ \\
\hline & High & 67 & $(74.4)$ \\
\hline BCL2 & Negative & 48 & $(53.3)$ \\
\hline & Positive & 42 & $(46.7)$ \\
\hline MYC & Negative & 58 & $(64.4)$ \\
\hline & Positive & 32 & $(35.6)$ \\
\hline Co-expression & Non-expressor & 43 & $(47.8)$ \\
\hline & BCL2 expressor & 15 & $(16.6)$ \\
\hline & MYC expressor & 5 & $(5.6)$ \\
\hline & Double expressor & 27 & $(30)$ \\
\hline Bouble hit & Absent & 83 & $(92.2)$ \\
\hline & Present & 7 & $(7.8)$ \\
\hline
\end{tabular}

abundant apoptosis, and frequent mitosis. All cases expressed CD20 and CD79a. All DHL patients showed CD10 positivity. In DEL, $85 \%$ was CD10 positive and
A

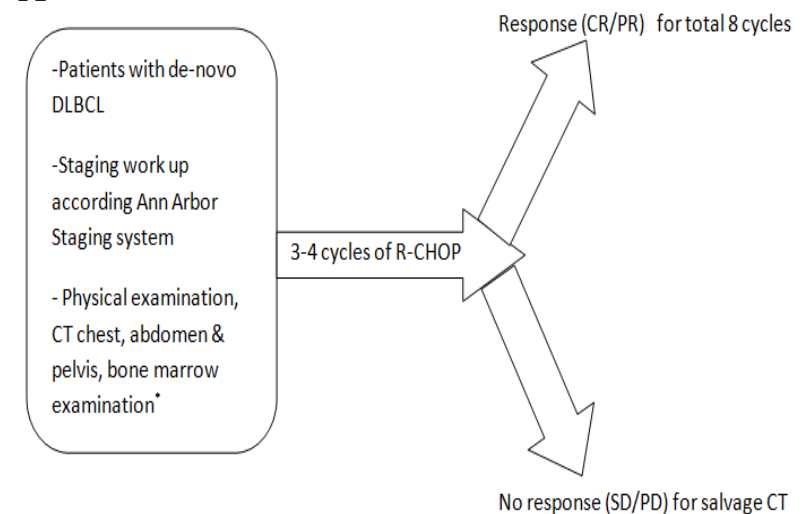

* Other investigations are symptoms and signs related; as lumbar puncture, MRI brain.

B

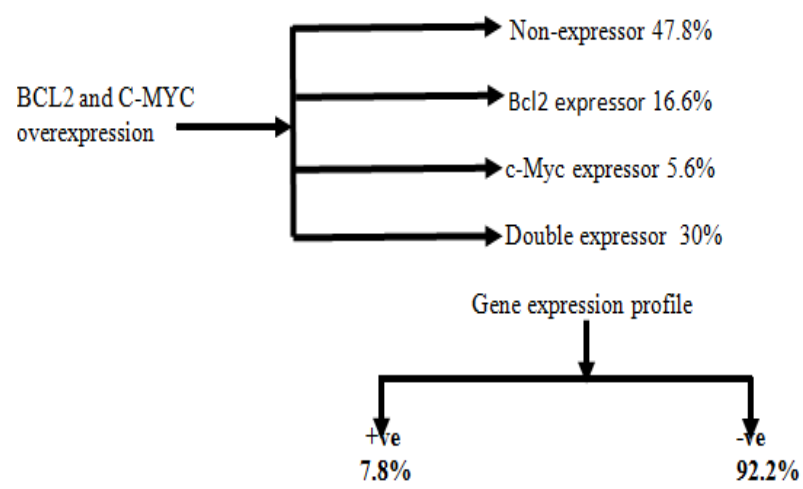

Figure 1. A, Treatment flow chart; B, Hans-algorithm for our patients; All cases express CD20 and CD79a. CD10 was $100 \%$ and $85 \%$ in double-hit and double-expressor lymphomas, respectively.

\section{$45 \%$ was BCL6 positive.}

Considering the treatment assessment, $22.2 \%$ of our patients achieved complete response (CR), 31.1\% had a partial response (PR), and $16.7 \%$ had stable disease (SD), while the remaining showed progressive disease (PD). The main patients' characteristics showed in Table 1 and Figure 1B.

The clinical-pathological features of DEL/DHL

$M Y C$ and $B C L 2$ proteins were detected in $35.6 \%$ and $46.7 \%$ of patients, respectively. Co-expression was present in $30 \%$. Most of the patients with DEL and DHL had an advanced stage (III, IV), intermediate to high IPI (P-value $<0.001)$, and more extra nodal involvement. Table2.

\section{The relation between DEL, DHL, and outcome}

Patients with $M Y C$ and BCL2 overexpression/gene translocation had a significantly poor outcome. All patients with DHL had DP while those with DEL showed PR and $\mathrm{SD}$ in $3.7 \%$ and $22.2 \%$, respectively. For DEL patients, the OS was 14 months and PFS was 2 months compared with DHL; the OS was 6 months (all patients with DHL died during the follow-up period). Table 3,4 and Figure 2, 3 . $m R N A$ levels of $M Y C$ and $B C L-2$ in patients with $D L B C L$

RT- PCR analysis showed that the $M Y C$ mRNA was $4.53 \pm 0.74$ and BCL-2 mRNA was $2.18 \pm 0.78$. 
Table 2. Relation between Clinical-pathological Features and DEL/DHL*

\begin{tabular}{|c|c|c|c|c|c|c|c|c|}
\hline \multirow[t]{3}{*}{ Characteristics } & \multicolumn{4}{|c|}{ BCL2/c-Myc Expression } & \multirow[t]{2}{*}{ p-value } & \multicolumn{2}{|c|}{ Double hit } & \multirow[t]{3}{*}{ p-value } \\
\hline & $\begin{array}{c}\text { Non- } \\
\text { expressor } \\
(\mathrm{N}=43)\end{array}$ & $\begin{array}{c}\text { BCL2 } \\
\text { expressor } \\
(\mathrm{N}=15)\end{array}$ & $\begin{array}{l}\text { c-Myc } \\
\text { expressor } \\
(\mathrm{N}=5)\end{array}$ & $\begin{array}{l}\text { Double } \\
\text { expressor } \\
(\mathrm{N}=27)\end{array}$ & & $\begin{array}{l}\text { Absent } \\
(\mathrm{N}=83)\end{array}$ & $\begin{array}{l}\text { Present } \\
(\mathrm{N}=7)\end{array}$ & \\
\hline & No.(\%) & No.(\%) & No.(\%) & No. $(\%)$ & & No.(\%) & No.(\%) & \\
\hline \multicolumn{9}{|l|}{ Age (years) } \\
\hline Mean \pm SD & $54.45 \pm 13.91$ & $53.5 \pm 12.94$ & $54.4 \pm 15.12$ & $66.18 \pm 11.78$ & $0.002 *$ & $57.1 \pm 14.12$ & $66.28 \pm 11.87$ & $0.099^{*}$ \\
\hline Median (Range) & $55(25-80)$ & $55.5(27-72)$ & $54(34-76)$ & $67(40-90)$ & & $57(25-90)$ & $67(45-79)$ & \\
\hline \multicolumn{9}{|l|}{ Sex } \\
\hline Male & $22(44.90 \%)$ & $9(18.30 \%)$ & $3(6.10 \%)$ & $15(30.60 \%)$ & $0.978 \ddagger$ & $44(89.80 \%)$ & $5(10.20 \%)$ & $0.448 \ddagger$ \\
\hline Female & $21(51.20 \%)$ & $6(14.60 \%)$ & $2(4.90 \%)$ & $12(29.30 \%)$ & & $39(95.10 \%)$ & $2(4.90 \%)$ & \\
\hline \multicolumn{9}{|c|}{ Extranodal involvement } \\
\hline Absent & $42(65.60 \%)$ & $9(14.00 \%)$ & $4(6.30 \%)$ & $9(14.10 \%)$ & $<0.001 \uparrow$ & $63(98.40 \%)$ & $1(1.60 \%)$ & $0.002 \ddagger$ \\
\hline Present & $1(3.80 \%)$ & $6(23.10 \%)$ & $1(3.80 \%)$ & $18(69.20 \%)$ & & $20(76.90 \%)$ & $6(23.10 \%)$ & \\
\hline \multicolumn{9}{|l|}{ Stage } \\
\hline Stage I & $12(85.70 \%)$ & $2(14.30 \%)$ & $0(0 \%)$ & $0(0 \%)$ & $<0.001 \S$ & $14(100 \%)$ & $0(0 \%)$ & $0.005 \S$ \\
\hline Stage II & $17(68 \%)$ & $4(16 \%)$ & $2(8 \%)$ & $2(8 \%)$ & & $25(100 \%)$ & $0(0 \%)$ & \\
\hline Stage III & $12(34.30 \%)$ & $9(25.70 \%)$ & $2(5.70 \%)$ & $12(34.30 \%)$ & & $32(91.40 \%)$ & $3(8.60 \%)$ & \\
\hline Stage IV & $2(12.50 \%)$ & $0(0 \%)$ & $1(6.30 \%)$ & $13(81.30 \%)$ & & $12(75 \%)$ & $4(25 \%)$ & \\
\hline \multicolumn{9}{|l|}{ IPI risk group } \\
\hline Low & $13(85.70 \%)$ & $2(14.30 \%)$ & $0(0 \%)$ & $0(0 \%)$ & $<0.001 \S$ & $14(100 \%)$ & $0(0 \%)$ & $<0.001 \S$ \\
\hline Low-Intermediate & $15(65.20 \%)$ & $4(17.40 \%)$ & $2(8.70 \%)$ & $2(8.70 \%)$ & & $23(100 \%)$ & $0(0 \%)$ & \\
\hline High-Intermediate & $14(42.40 \%)$ & $8(24.20 \%)$ & $2(6.10 \%)$ & $9(27.30 \%)$ & & $33(100 \%)$ & $0(0 \%)$ & \\
\hline High & $2(10 \%)$ & $1(5 \%)$ & $1(5 \%)$ & $16(80 \%)$ & & $13(65 \%)$ & $7(35 \%)$ & \\
\hline \multicolumn{9}{|l|}{ ki67 } \\
\hline Low & $22(95.70 \%)$ & $1(4.30 \%)$ & $0(0 \%)$ & $0(0 \%)$ & $<0.001 \$$ & $23(100 \%)$ & $0(0 \%)$ & $0.184 \ddagger$ \\
\hline High & $21(31.30 \%)$ & $14(20.90 \%)$ & $5(7.50 \%)$ & $27(40.30 \%)$ & & $60(89.60 \%)$ & $7(10.40 \%)$ & \\
\hline
\end{tabular}

Double expressor/double hit lymphomas*

The quantitative expressions of $M Y C$ and $B C L-2$ in tissue were significantly higher in DLBCL patients compared to the control $(\mathrm{P}<0.001)$. There was a statistically significant correlation between protein overexpression and mRNA of both MYC and BCL2 $(\mathrm{p}<0.001)$ Table5.

\section{Discussion}

DLBCL is the most common NHL, representing approximately $40 \%$ of all lymphoma all over the world. It is a heterogeneous disease with multiple biological distinct disorders. In the recent WHO revision of lymphoma classification and based on $G E P$, a new category is recognized as "high-grade B-cell lymphoma ( $H G B L)$
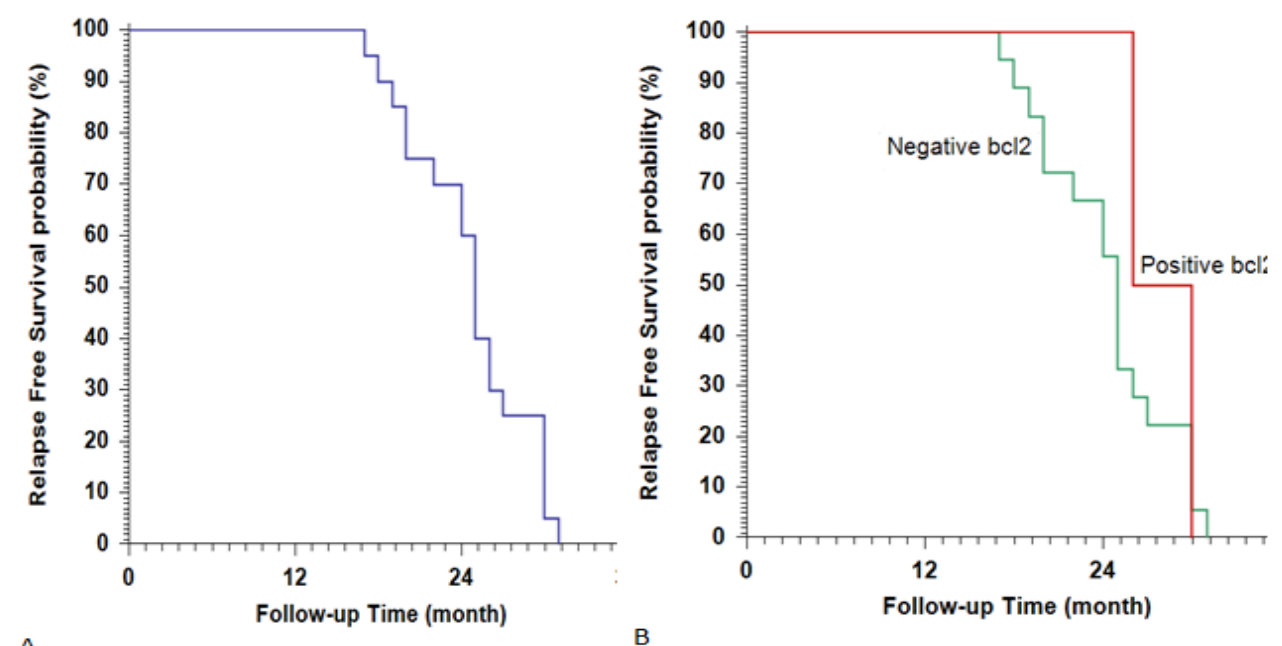

Figure 2. Kaplan-Meier Plot of Relapse Free Survival (RFS), (A) All studied patients; (B) stratified according to BCL2.

1466 Asian Pacific Journal of Cancer Prevention, Vol 20 
DOI:10.31557/APJCP.2019.20.5.1463

C-MYC and BCL2 in Diffuse Large B Cell Lymphoma

Table 3. Relation between Immunohistochemical Markers and Outcome

\begin{tabular}{|c|c|c|c|c|c|c|c|c|c|}
\hline \multirow[t]{3}{*}{ Outcome } & \multicolumn{2}{|c|}{$\mathrm{Ki67}$} & \multirow[t]{3}{*}{ p-value } & \multicolumn{2}{|c|}{ BCL2 } & \multirow[t]{3}{*}{ p-value } & \multicolumn{2}{|c|}{ c-Myc } & \multirow[t]{3}{*}{ p-value } \\
\hline & $\begin{array}{c}\text { Low } \\
(\mathrm{N}=23)\end{array}$ & $\begin{array}{c}\text { High } \\
(\mathrm{N}=67)\end{array}$ & & $\begin{array}{l}\text { Negative } \\
(\mathrm{N}=48)\end{array}$ & $\begin{array}{l}\text { Positive } \\
(\mathrm{N}=42)\end{array}$ & & $\begin{array}{l}\text { Negative } \\
(\mathrm{N}=58)\end{array}$ & $\begin{array}{l}\text { Positive } \\
(\mathrm{N}=32)\end{array}$ & \\
\hline & No. $(\%)$ & No. $(\%)$ & & No. $(\%)$ & No. $(\%)$ & & No. $(\%)$ & No. $(\%)$ & \\
\hline \multicolumn{10}{|l|}{ Response } \\
\hline $\mathrm{PD}$ & $1(4.3 \%)$ & $26(38.8 \%)$ & $<0.001 \ddagger$ & $3(6.3 \%)$ & $24(57.1 \%)$ & $<0.001 \ddagger$ & $4(6.9 \%)$ & $23(71.9 \%)$ & $<0.001$; \\
\hline SD & $1(4.3 \%)$ & $14(20.9 \%)$ & & $3(6.3 \%)$ & $12(28.6 \%)$ & & $8(13.8 \%)$ & $7(21.9 \%)$ & \\
\hline PR & $15(65.2 \%)$ & $13(19.4 \%)$ & & $24(50 \%)$ & $4(9.5 \%)$ & & $26(44.8 \%)$ & $2(6.3 \%)$ & \\
\hline $\mathrm{CR}$ & $6(26.1 \%)$ & $14(20.9 \%)$ & & $18(37.5 \%)$ & $2(4.8 \%)$ & & $20(34.5 \%)$ & $0(0 \%)$ & \\
\hline NR & $2(8.7 \%)$ & $40(59.7 \%)$ & $<0.001 \ddagger$ & $6(12.5 \%)$ & $36(85.7 \%)$ & $<0.001$ & $12(20.7 \%)$ & $30(93.8 \%)$ & $<0.001 \ddagger$ \\
\hline OAR & $21(91.3 \%)$ & $27(40.3 \%)$ & & $42(87.5 \%)$ & $6(14.3 \%)$ & & $46(79.3 \%)$ & $2(6.3 \%)$ & \\
\hline Relapse & $(\mathrm{N}=6)$ & $(\mathrm{N}=14)$ & & $(\mathrm{N}=18)$ & $(\mathrm{N}=2)$ & & $(\mathrm{N}=20)$ & & \\
\hline Before 24 month & $4(66.7 \%)$ & $4(28.6 \%)$ & $0.161 \ddagger$ & $8(44.4 \%)$ & $0(0 \%)$ & $0.495 \ddagger$ & $8(40 \%)$ & & --- \\
\hline After 24 month & $2(33.3 \%)$ & $10(71.4 \%)$ & & $10(55.6 \%)$ & $2(100 \%)$ & & $12(60 \%)$ & & \\
\hline \multicolumn{10}{|l|}{ RFS } \\
\hline Mean RFS & 22.17 month & 25.79 month & $0.020 \dagger$ & 24.33 month & 28 month & $0.416 \dagger$ & 24.70 month & & \\
\hline$(95 \% \mathrm{CI})$ & $(19.62-24.72)$ & $(23.51-28.06)$ & & $(22.34-26.33)$ & $(24.08-31.92)$ & & $(22.82-26.58)$ & & \\
\hline Median RFS & 22 month & 26 month & & 25 month & 26 month & & 25 month & & \\
\hline 24 month RFS & $33.3 \%$ & $71.4 \%$ & & $55.6 \%$ & $100 \%$ & & $60 \%$ & & \\
\hline 30 month RFS & $0 \%$ & $7.1 \%$ & & $5.6 \%$ & $0 \%$ & & $5 \%$ & & \\
\hline \multicolumn{10}{|l|}{ PFS } \\
\hline Progression & $(\mathrm{N}=16)$ & $(\mathrm{N}=26)$ & & $(\mathrm{N}=27)$ & $(\mathrm{N}=15)$ & & $(\mathrm{N}=33)$ & $(\mathrm{N}=9)$ & \\
\hline Within 6 monh & $9(56.3 \%)$ & $21(80.8 \%)$ & $0.158 \ddagger$ & $15(55.6 \%)$ & $15(100 \%)$ & $0.003 t$ & $21(63.6 \%)$ & $9(100 \%)$ & $0.041 \%$ \\
\hline After 6 month & $7(43.8 \%)$ & $5(19.2 \%)$ & & $12(44.4 \%)$ & $0(0 \%)$ & & $12(36.4 \%)$ & $0(0 \%)$ & \\
\hline \multicolumn{10}{|l|}{ PFS } \\
\hline $\begin{array}{l}\text { Mean PFS } \\
(95 \% \mathrm{CI})\end{array}$ & $\begin{array}{l}6.38 \text { months } \\
(5.66-7.09)\end{array}$ & $\begin{array}{l}4.15 \text { months } \\
(3.15-5.15)\end{array}$ & $0.039 \dagger$ & $\begin{array}{l}6.37 \text { months } \\
(5.66-7.08)\end{array}$ & $\begin{array}{c}2.53 \text { months } \\
(2-3.07)\end{array}$ & $<0.001 \dagger$ & $\begin{array}{l}5.82 \text { months } \\
(5.11-6.53)\end{array}$ & $\begin{array}{c}2 \text { months } \\
(1.35-2.62)\end{array}$ & $<0.001 \dagger$ \\
\hline Median PFS & 6 months & 3 months & & 6 months & 3 months & & 6 months & 2 months & \\
\hline 3 month PFS & $100 \%$ & $50 \%$ & & $96.3 \%$ & $20 \%$ & & $84.9 \%$ & $11.1 \%$ & \\
\hline 6 month PFS & $43.8 \%$ & $19.2 \%$ & & $44.4 \%$ & $0 \%$ & & $36.3 \%$ & $0 \%$ & \\
\hline 9 month PFS & $6.3 \%$ & $3.9 \%$ & & $7.4 \%$ & $0 \%$ & & $6.1 \%$ & $0 \%$ & \\
\hline \multicolumn{10}{|l|}{ Mortality } \\
\hline Alive & $23(100 \%)$ & $43(64.2 \%)$ & $0.001 \ddagger$ & $46(95.8 \%)$ & $20(47.6 \%)$ & $<0.001 \$$ & $56(96.6 \%)$ & $10(31.3 \%)$ & $<0.001 \%$ \\
\hline Died & $0(0 \%)$ & $24(35.8 \%)$ & & $2(4.2 \%)$ & $22(52.4 \%)$ & & $2(3.4 \%)$ & $22(68.8 \%)$ & \\
\hline \multicolumn{10}{|l|}{ OS } \\
\hline $\begin{array}{l}\text { Mean OS } \\
(95 \% \mathrm{CI})\end{array}$ & 36 month & $\begin{array}{c}27.37 \text { month } \\
(24.54-30.19)\end{array}$ & $0.001 \dagger$ & $\begin{array}{c}35.17 \text { month } \\
(34.01-36.32)\end{array}$ & $\begin{array}{c}21.58 \text { month } \\
(18.22-24.94)\end{array}$ & $<0.001 \dagger$ & $\begin{array}{c}35.33 \text { month } \\
(34.40-36.26)\end{array}$ & $\begin{array}{c}17.84 \text { month } \\
(13.95-21.73)\end{array}$ & $<0.001 \dagger$ \\
\hline Median OS & NR & NR & & NR & 20 month & & NR & 15 month & \\
\hline 24 month OS & $100 \%$ & $63 \%$ & & $95.8 \%$ & $44 \%$ & & $96.6 \%$ & $22.1 \%$ & \\
\hline 30 month OS & $100 \%$ & $63 \%$ & & $95.8 \%$ & $44 \%$ & & $96.6 \%$ & $22.1 \%$ & \\
\hline
\end{tabular}

with $M Y C$ and $B C L 2$ and/or BCL6 rearrangements, with removal the category of unclassified lymphoma (Li et al., 2018).

$M Y C$ is a transcription factor located on chromosome 8 (8q24), regulates the expression of several target genes involved in DNA damage and repair. Cells with MYC translocations usually have TP53 mutations allowing them to escape apoptosis and survive (Sehn et al., 2005; Barrans et al., 2010). BCL2 and $B C L 6$ are anti-apoptotic factors deregulated in $D L B C L$ via chromosomal translocation or gene rearrangement (Swerdlow et al., 2008; Lenz et al., 2008). Ki67 is usually associated with the advanced/aggressive disease however; its impact on survival outcome is controversy (Miller et al., 1994; Bryant et al., 2006).
In our study and among DEL patients, the morphological pattern of diffuse growth was the commonest (99\%) with a "starry sky" pattern of $20 \%$ of the cases, which represented the main features of BL. In the DHL group, the typical morphology was a diffuse monomorphic pattern of numerous apoptotic bodies (Oliveira et al., 2017).

Most of our patients with DEL or DHL had a high stage (III, IV), intermediate to high IPI, and higher extra- node involvement $(\mathrm{P}<0.001)$. These results matched with many previous studies (Oliveira et al., 2017; Snuderl et al., 2010; Riedell et al., 2018; Friedberg, 2017, Reagan et al., 2017).

Moreover, protein overexpression of $M Y C, B C L 2$, and co-expression was detected in $35.6 \%, 46.7 \%$, and $30 \%$ of patients, respectively. $7.8 \%$ of our patients were 
Table 4. Relation between DEL and DHL

\begin{tabular}{|c|c|c|c|c|c|c|c|c|}
\hline \multirow[t]{3}{*}{ Outcome } & \multicolumn{4}{|c|}{ BCL2/c-Myc Expression } & \multicolumn{3}{|c|}{ Double hit } & \multirow[t]{3}{*}{$\mathrm{p}$-value } \\
\hline & $\begin{array}{c}\text { Non-expressor } \\
(\mathrm{N}=43)\end{array}$ & $\begin{array}{c}\text { BCL2 expressor } \\
\qquad(\mathrm{N}=15)\end{array}$ & $\begin{array}{l}\text { c-Myc expressor } \\
\quad(\mathrm{N}=5)\end{array}$ & $\begin{array}{c}\text { Double expressor } \\
\qquad(\mathrm{N}=27)\end{array}$ & p-value & $\begin{array}{l}\text { Absent } \\
(\mathrm{N}=83)\end{array}$ & $\begin{array}{l}\text { Present } \\
(\mathrm{N}=7)\end{array}$ & \\
\hline & No. $(\%)$ & No. $(\%)$ & No. (\%) & No.(\%) & & No. (\%) & No. (\%) & \\
\hline \multicolumn{9}{|l|}{ Response } \\
\hline PD & $0(0.0 \%)$ & $4(26.7 \%)$ & $3(60 \%)$ & $20(74.1 \%)$ & $<0.001 \S$ & 20 (24.1\%) & $7(100 \%)$ & $0.001 \ddagger$ \\
\hline SD & $2(4.6 \%)$ & $6(40.0 \%)$ & $1(20 \%)$ & $6(22.2 \%)$ & & $15(18.1 \%)$ & $0(0 \%)$ & \\
\hline PR & $23(53.5 \%)$ & $3(20.0 \%)$ & $1(20 \%)$ & $1(3.7 \%)$ & & $28(33.7 \%)$ & $0(0 \%)$ & \\
\hline $\mathrm{CR}$ & $18(41.9 \%)$ & $2(13.3 \%)$ & $0(0 \%)$ & $0(0 \%)$ & & $20(24.1 \%)$ & $0(0 \%)$ & \\
\hline NR & $2(4.7 \%)$ & $10(66.7 \%)$ & $4(80 \%)$ & $26(96.3 \%)$ & $<0.001 \S$ & $35(42.2 \%)$ & $7(100 \%)$ & $0.004 \%$ \\
\hline OAR & $41(95.3 \%)$ & $5(33.3 \%)$ & $1(20 \%)$ & $1(3.7 \%)$ & & $48(57.8 \%)$ & $0(0 \%)$ & \\
\hline Relapse & $(\mathrm{N}=18)$ & $(\mathrm{N}=2)$ & & & & $(\mathrm{N}=20)$ & & \\
\hline Before 24 month & $8(44.4 \%)$ & $0(0 \%)$ & & & $0.495 \$$ & $8(40 \%)$ & & --- \\
\hline After 24 month & $10(55.6 \%)$ & $2(100 \%)$ & & & & $12(60 \%)$ & & \\
\hline \multicolumn{9}{|l|}{ RFS } \\
\hline Mean RFS & 24.33 month & 28 month & & & $0.416 \dagger$ & 24.70 month & & \\
\hline$(95 \% \mathrm{CI})$ & $(22.34-26.33)$ & $(24.08-31.92)$ & & & & $(22.82-26.58)$ & & \\
\hline Median RFS & 25 month & 26 month & & & & 25 month & & \\
\hline 24 month RFS & $55.6 \%$ & $100 \%$ & & & & $60 \%$ & & \\
\hline 30 month RFS & $5.6 \%$ & $0 \%$ & & & & $5 \%$ & & \\
\hline Progression & $(\mathrm{N}=25)$ & $(\mathrm{N}=8)$ & $(\mathrm{N}=2)$ & $(\mathrm{N}=7)$ & & & & \\
\hline Within 6month & $13(52 \%)$ & $8(100 \%)$ & $2(100 \%)$ & $7(100 \%)$ & $0.005 \S$ & & & \\
\hline After 6 month & $12(48 \%)$ & $0(0 \%($ & $0(0 \%)$ & $0(0 \%)$ & & & & \\
\hline \multicolumn{9}{|l|}{ PFS } \\
\hline Mean PFS & 6.60 months & 3.38 months & 3.50 months & 1.57 months & $<0.001 \dagger$ & & & \\
\hline$(95 \% \mathrm{CI})$ & $(5.91-7.29)$ & $(3.02-3.373)$ & $(2.52-4.48)$ & $(1.18-1.97)$ & & & & \\
\hline Median PFS & 6 months & 3 months & 3 months & 2 months & & & & \\
\hline 3 month PFS & $100 \%$ & $37.5 \%$ & $50 \%$ & $0 \%$ & & & & \\
\hline 6 month PFS & $48 \%$ & $0 \%$ & $0 \%$ & $0 \%$ & & & & \\
\hline 9 month PFS & $8 \%$ & $0 \%$ & $0 \%$ & $0 \%$ & & & & \\
\hline \multicolumn{9}{|l|}{ Mortality } \\
\hline Alive & $43(100 \%)$ & $13(86.7 \%)$ & $3(60 \%)$ & $7(25.9 \%)$ & $<0.001 \S$ & $66(79.5 \%)$ & $0(0 \%)$ & $<0.001$ \\
\hline Died & $0(0 \%)$ & $2(13.3 \%)$ & $2(40 \%)$ & $20(74.1 \%)$ & & $17(20.5 \%)$ & $7(-100 \%)$ & \\
\hline \multicolumn{9}{|l|}{ OS } \\
\hline Mean OS & 36 month & 30.64 month & 26.40 month & 14.95 month & $<0.001 \dagger$ & 31.57 month & 6.57 month & $<0.001 \dagger$ \\
\hline$(95 \% \mathrm{CI})$ & & $(27.54-33.75)$ & $(17.41-35.39)$ & (11.93-17.96) & & $(29.68-33.46)$ & $(4.98-8.16)$ & \\
\hline Median OS & & NR & 35 month & 14 month & & 36 month & 6 month & \\
\hline 24 month OS & $100 \%$ & $85.7 \%$ & $53.3 \%$ & $12.4 \%$ & & $78.9 \%$ & $0 \%$ & \\
\hline 30 month OS & $100 \%$ & $85.7 \%$ & $53.3 \%$ & $12.4 \%$ & & $78.9 \%$ & $0 \%$ & \\
\hline
\end{tabular}

Double expressor/double hit lymphomas*

Table 5. The Relation between mRNA MYC, BCL-2 Levels and their Protein Expression

\begin{tabular}{lcc}
\hline & mRNA Myc levels & $\mathrm{p}$ \\
\hline +veMyc protein expression $(\mathrm{n}=32)$ & $4.53 \pm 0.74$ & $<0.001$ \\
- veMyc protein expression $(\mathrm{n}=58)$ & $0.95 \pm 0.41$ & $<0.001$ \\
& mRNA BCL-2 levels & \\
+veBCL-2 protein expression $(\mathrm{n}=42)$ & $2.18 \pm 0.78$ & $0.88 \pm 0.11$ \\
- veBCL-2 protein expression $(\mathrm{n}=48)$ & & \\
\hline
\end{tabular}

DHL identified by RT-PCR, which inconsistent with many previous data. In a retrospective study done by Green et al., (2012) on 193 newly diagnosed DLBCL to evaluate $M Y C$ and $B C L 2$ using $I H C$ and $F I S H$ revealed that $6 \%$ of patients were diagnosed as $D H L$ by $F I S H$ and $29 \%$ as
DEL using IHC. In addition, Yan et al., (2014) in another retrospective study on 336 patients of de novo DLBCL treated with $\mathrm{CHOP} \pm \mathrm{R}$ to evaluate the prognostic value of MYC, BCL2, BCL6 using IHC or FISH. The results showed that protein overexpression of $M Y C, B C L 2$, and BCL6 
A

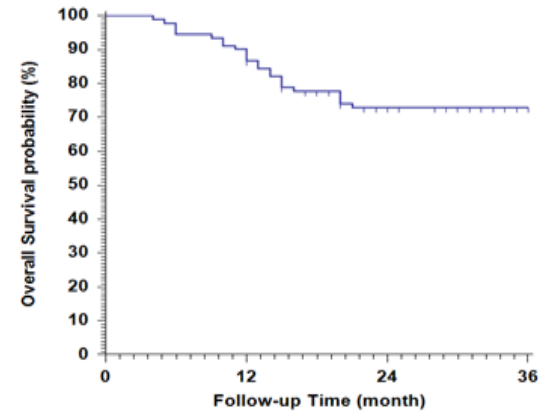

B

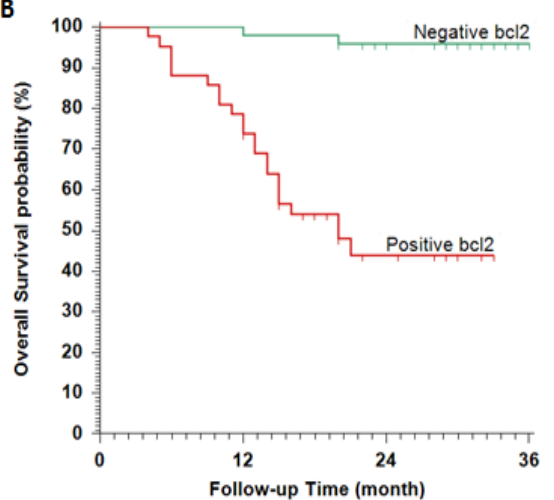

D

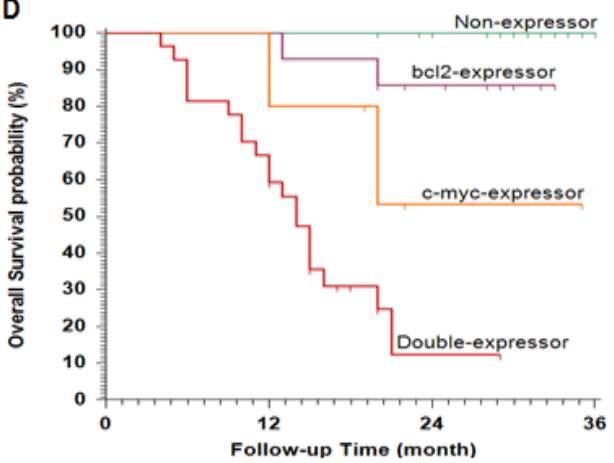

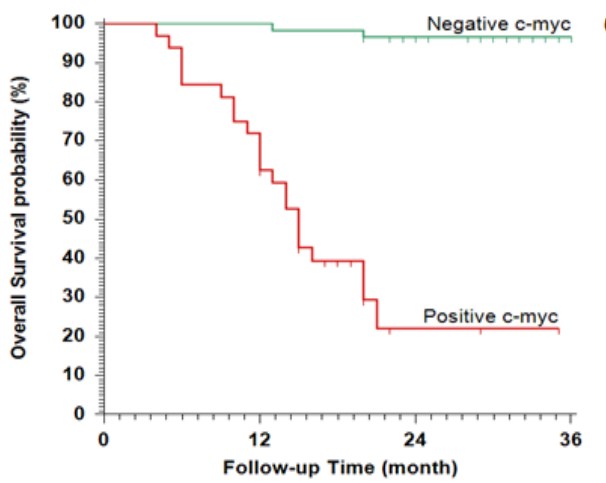

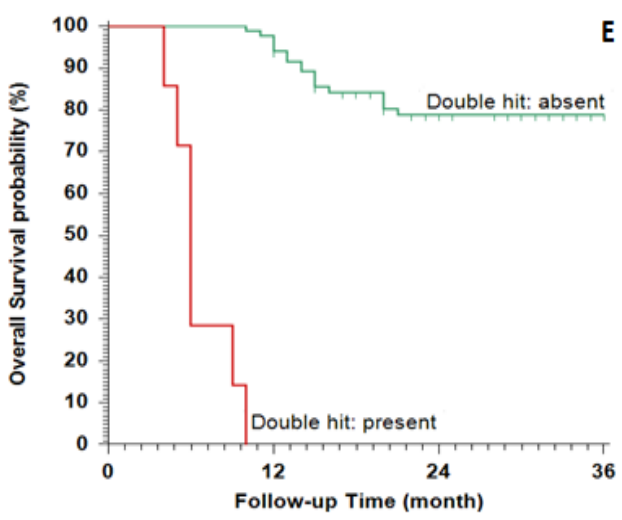

Figure 3. Kaplan-Meier Plot of Overall Survival (OS), (A), All studied patients; (B), stratified according to BCL2; (C), stratified according to c-Myc expression; (D), stratified according to BCl2/c-Myc expression; (E), stratified according to double hit.

were $\geq 40 \%, \geq 70 \%$, and $\geq 50 \%$, respectively.

In the present study, the median OS are 6 months for DHL patients compared with 36 months in the absence of the translocation. These results are corresponding to the previous study done on 57 patients with high-grade NHL revealed shorter median OS for DHL compared with non-DHL (8.2 vs. 56.8 months, $\mathrm{P}<0.001$ ) (Lands burg et al., 2014). Moreover, all our patients with DHL died through our study. In another retrospective study done on 120 Brazilian patients with aggressive NHL by Oliveira et al., (2014), reported a poor outcome associated with DHL where most of the patients died within 6 months.

2-year OS for DEL was 14 months. Similar findings were reported by Savage et al., (2009) when evaluated the outcome of 135 patients with DLBCL post-treatment with R-CHOP. The 5-year OS was significantly worse in MYC positive $(33 \%)$, with a high incidence of $\mathrm{CNS}$ relapse, compared with $M Y C$ negative cases $(72 \%)$. Another retrospective studied on 69 eligible patients, Aggarwal et al., (2016) reported poor outcome for patients with DEL compared with non-DEL.

Through the measuring of the cell proliferation rate by the percentage of Ki67 labelling index, as a selective parameter of DLBCL patients for further cytogenetic tests is an area of controversy. A study done by Kalaw et al., (2012) concluded that $90 \%$ as a cut-off value was not indicative for $M Y C$ translocations.

Hence, DHL is a temporary title for aggressive B-cell lymphomas, diagnosed not only by a unique aggressive clinical course associated with disease progression but also by its gene rearrangements. Those patients require a new treatment regimen based on gene repression of possible genetic mechanisms, or certain protocol that may eliminate the effect of the cytogenetic aberrations.

Meanwhile, our results showed a statistically significant association with protein overexpression detected by IHC and gene translocation identified by mRNA for both $M Y C$ and BCL2. Owing to this high specificity between IHC and cytogenetic tests by FISH, the economic problems with developing countries, and 
the importance of risk stratification, can we replace IHC instead of GEP?

\section{Conclusions and Recommendation}

Our findings confirm that regardless of the way of detection of MYC and BCL2 either by IHC or FISH, they associated with unique pathological features (high mitotic rates and starry sky appearance) and poor outcome. Because the IHC is a conventional and accessible method of assessment in the developing countries like Egypt, it may replace the cytogenetic study and can act as selection criteria for further cytogenetic testing. Regarding the unsatisfying response, still, the era of treatment of DLBCL is attractive to further research. Based on the progress of molecular findings, tailored therapy or risk-adapted therapy is mostly the best moving forward.

\section{Limitations}

The retrospective study depends totally on documentation by medical staff, so it is almost always criticized due to insufficient data. Also, the small sample size may represent an obstacle to get clearer and power data.

\section{Conflict of interest}

The authors certify that there is no potential or actual conflict of interest related to this research.

\section{References}

Aggarwal A, Rafei H, Alakeel F, et al (2016).Outcome of patients with double-expressor lymphomas (DELs) treated with R-CHOP or R-EPOCH. Blood, 128, 5396.

Aukema SM, Siebert R, Schuuring E, et al (2011). Double-hit B-cell lymphomas. Blood, 117, 2319-31.

Barrans S, Crouch S, Smith A, et al (2010). Rearrangement of $M Y C$ is associated with poor prognosis in patients with diffuse large B-cell lymphoma treated in the era of rituximab. J Clin Oncol, 28, 3360-5.

Barrans SL, Crouch S, Care MA, et al (2012). Whole genome expression profiling based on paraffin embedded tissue can be used to classify diffuse large B-cell lymphoma and predict clinical outcome. Br J Haematol, 159, 441-53.

Bryant RJ, Banks PM, O’Malley DP (2006). Ki-67 staining pattern as a diagnostic tool in the evaluation of lymphoproliferative disorders. Histopathology, 48, 505-15.

Cheah CY, Oki Y, Westin JR, et al (2015). A clinician's guide to double hit lymphomas. Br J Haematol, 168, 784-95.

Friedberg JW (2017). How I treat double-hit lymphoma. Blood, 130, 590-6.

Green TM, Young KH, Visco C, et al (2012). Immunohistochemical double-hit score is a strong predictor of outcome in patients with diffuse large B-cell lymphoma treated with rituximab plus cyclophosphamide, doxorubicin, vincristine, and prednisone. J Clin Oncol, 30, 3460-7.

Hu S, Xu-Monette ZY, Tzankov A, et al (2013). MYC/BCL2 protein coexpression contributes to the inferior survival of activated B-cell subtype of diffuse large B-cell lymphoma and demonstrates high-risk gene expression signatures: A report from The International DLBCL Rituximab-CHOP Consortium Program. Blood, 121, 4021-31.

Jaffe E, Harris NL, Stein H, et al (2008). Classification of lymphoid neoplasms: the microscope as a tool for disease discovery. Blood, 112, 4384-6.
Lands burg DJ, Nasta SD, Svoboda J, et al (2014). Double-hit cytogenetic status may not be predicted by baseline clinicopathological characteristics and is highly associated with overall survival in B cell lymphoma patients. Br J Hematol, 166, 169-74.

Lenz G, Wright G, Dave SS, et al (2008): Stromal gene signatures in large-B-cell lymphomas. N Engl J Med, 359, 2313-23.

Li S, Young KH, Medeiros LJ (2018). Diffuse large B-cell lymphoma. Pathology, 50, 74-87.

Livak KJ, Schmittgen TD, (2001). Analysis of relative gene expression data using real time quantitative PCR and the $2^{-\Delta \Delta C \mathrm{~T}}$ method. Methods, 25, 402-8.

Mation-Kalaw E, Tan LHC, Tay K, et al (2012). Does the proliferation fraction help identify mature $\mathrm{B}$ cell lymphomas with double- and triple-hit translocations?. Histopathology, 61, 1214-8.

Miller TP, Grogan TM, Dahlberg S, et al (1994). Prognostic significance of the Ki-67-associated proliferative antigen in aggressive non-Hodgkin's lymphomas: a prospective Southwest Oncology Group trial. Blood, 8, 1460-6.

Oliveira CC, Maciel-Guerra H, Kucko L (2017). Double-hit lymphomas: clinical, morphological, immunohistochemical and cytogenetic study in a series of Brazilian patients with high-grade non-Hodgkin lymphoma. Diagn Pathol, 12, 3.

Reagan PM, Davies A (2017). Current treatment of double hit and double expressor lymphoma. Hematol Am Soc Hematol Educ Program, 8, 295-7.

Riedell PA, Smith SM (2018). Double hit and double expressors in lymphoma: Definition and treatment. Cancer, 15, 4622-32.

Savage KJ, Johnson NA, Ben-Neriah S, et al (2009). MYC gene rearrangements are associated with a poor prognosis in diffuse large B-cell lymphoma patients treated with R-CHOP chemotherapy. Blood, 114, 3533-7.

Sehn LH, Donaldson J, Chhanabhai M, et al (2005). Introduction of combined CHOP plus rituximab therapy dramatically improved outcome of diffuse large B-cell lymphoma in British Columbia. J Clin Oncol, 23, 5027-33.

Snuderl M, Kolman OK, Chen YB, et al (2010). B-cell lymphomas with concurrent $I G H-B C L 2$ and $M Y C$ rearrangements are aggressive neoplasms with clinical and pathologic features distinct from Burkitt lymphoma and diffuse large B-cell lymphoma. Am J Surg Pathol, 34, $327-40$.

Swerdlow SH, Campo E, Pileri SA, et al (2016). The 2016 revision of the World Health Organization classification of lymphoid neoplasms. Blood, 127, 2375-90.

Swerdlow SH, Campo E, Pileri SA, et al (2016). The 2016 revision of the World Health Organization classification of lymphoid neoplasms. Blood, 127, 2375-90.

Tang YL, Zhou Y, Cheng LL, et al (2017). BCL2/Ki-67 index predict survival in germinal center B-cell-like diffuse large B-cell lymphoma. Oncol Lett, 14, 3767-73.

Xia B, Zhang L, Guo SQ, et al (2015). Coexpression of MYC and $B C L-2$ predicts prognosis in primary gastrointestinal diffuse large B-cell lymphoma. World, 21, 2433-42.

Yan L-X, Liu Y-H, Luo D-L, et al (2014). MYC expression in concert with $B C L 2$ and $B C L 6$ expression predictis outcome in Chinese patients with diffuse large B-cell lymphoma, not otherwise specified. PLoS One, 9, e104068.

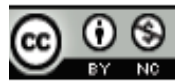

This work is licensed under a Creative Commons AttributionNon Commercial 4.0 International License. 